\title{
Utilizing a new approach for solving fully fuzzy linear programming problems
}

\author{
Hamiden Abdelwahed Khalifa* \\ Department of Operations Research, Institute of Statistical \\ Studies and Research, Cairo University, Giza, Egypt \\ Present Address: Mathematics Department, College of Science and Arts \\ Al-Badaya, Qassim University, Saudi Arabia \\ E-mail:〈hamidenkhalifa@cu.edu.eg,Ha.Ahmed@qu.edu.sa〉
}

\begin{abstract}
This paper deals with fully fuzzy linear programming (FFLP) problem in which all parameters and variables are characterized by $L-R$ fuzzy numbers. By a proposed approach, the FFLP problem is converted into the triple objective functions, and hence a single objective using the weighting method. Through this approach the problem is not transformed into the crisp linear programming problem (LPP) that is enable for obtaining fuzzy optimal solution and the corresponding fuzzy optimal solution which is more realistic to the real world problems. Then a numerical example is taken to the utility and clarify the practically and the efficiency of the approach.
\end{abstract}

Keywords: fully fuzzy linear programming, fuzzy optimal solution, $L-R$ fuzzy numbers, software GAMS, weighting method

Received: November 12, 2018; accepted: October 26, 2019; available online: December 13, 2019

DOI: $10.17535 /$ crorr.2019.0027

\section{Introduction}

Linear programming (LP) is a branch of mathematical programming designed to solve optimization problem for which all the constraints and objective functions are linear functions. LP is an approach for finding the answer fittest from a range of possible answers. LP problems have enormous applications as in arising in business, government, industry, hospitals, libraries, etc. It is clear that LP is in two forms: Classical LP and Fuzzy LP. Bector and Chandra [2] classified the fuzzy LP problems into four categories:

- Type I. LP with fuzzy inequalities and crisp objective function,

- Type II. LP with crisp inequalities and fuzzy objective function,

- Type III. LP with fuzzy inequalities and fuzzy objective function and

- Type IV. LP with fuzzy parameters.

In many scientific areas, such as system analysis and operations research, a model has to be set up using data which is only approximately known. Fuzzy sets theory, introduced by Zadeh [30], makes this possible. Dubois and Prade [10] extended the use of algebraic operations on real numbers to fuzzy numbers by the use a fuzzification principle.

${ }^{*}$ Corresponding author. 
Since the pioneer work on fuzzy LP by Tanaka et al. [28] and Zimmermann [33]. Several kinds of fuzzy LP problems of satisfying different constraints were appeared in the literature so far and with them corresponding approaches of resolution have proposed too. Tanaka et al. [29] formulated a fuzzy linear programming (FLP) problem to obtain a reasonable solution under consideration of the ambiguity of parameters. Zhao et al. [31] studied the complete solution set for fuzzy linear programming problems included fuzzy and non-fuzzy equality and inequality constraints. The basic concepts of fuzzy decision making in fuzzy environment were first proposed by Bellman and Zadeh [3] The first formulation of fuzzy LP problems was proposed by Zimmermann [33], Shaocheng [27], Buckley [4, 5] and others considered situation where all parameters are fuzzy. Li and Shi [18] studied fuzzy LP problems with interval- value fuzzy coefficients, where corresponding auxiliary models in different criteria are obtained. Zhong et al. [32] studied LP problem with fuzzy random variable coefficients and fuzzy pseudorandom variables. Maleki et al. [19] proposed a new method for solving LP problem with fuzzy variables based on the concept of comparison of fuzzy numbers. The references $[6,7,8,21,24,28]$ studied fuzzy mathematical programming with fuzzy number coefficients. Ishibuchi and Tanaka [15] investigated mathematical programming problem with interval objective function coefficients.

The fuzzy LP problems in which all the parameters as well as variables are represented by fuzzy numbers is known as FFLP problems. FFLP has many different applications in sciences and engineering, and various methods have been proposed for solving it. FFLP problems can be divided into two categories: FFLP with equality constraints and with equality constraints. Kumar et al. [17] proposed a new method for solving fully fuzzy LP problem with equality constraints and determined the optimal fuzzy solution for it. Hashemi et al. [13] and Allahviranloo et al. [1] proposed different methods for FFLP problems with inequality constraints. Sahaya Sudha and Karpagamani [23] discussed FFLP problem with trapezoidal fuzzy numbers with the help of linear system and ranking function. Rajarajeswari and Sahaya Sudha [22] proposed a new method for solving FFLP problem. Based on Lexicography method, Shamooshaki et al. [26] proposed a new method for solving FFLP problem. Hosseinzadeh and Edalatpanah [14] proposed a new method for solving FFLP based on the $L-R$ fuzzy numbers and the Lexicography method. Das [8] developed a modified algorithm to find the fuzzy optimal solution for the FFLP problem with equality constraints. Through the MOLP problem and extended L-R fuzzy numbers, Gong et al. [12] and Ezzati et al. [11] solved FFLP problems. Das et al. [9] used the ranking function for solving FFLP problem with mixed constraints so as to overcome limitations.

The rest of the paper is organized as follows. In Section 2, some preliminaries need in the paper are introduced. In Section 3, a fully fuzzy linear programming problem is formulated. In Section 4, a method for solving the FFLP problem is proposed. In Section 5, a numerical example is given for illustration. Finally, some concluding remarks are reported in Section 6.

\section{Preliminaries}

In order to discuss our problem conveniently, basic concepts and results related to fuzzy numbers, L-R fuzzy numbers and their arithmetic operations are recalled (Kaufmann and Gupta [16], Bellman and Zadeh [3], Sakawa [25] and Hosseinzadeh and Edalatpanah [14]).

Definition 1. A fuzzy number $\tilde{a}$ is mapping $\mu_{\tilde{a}}: R \rightarrow[0,1]$ with following properties:

i) $\mu_{\tilde{a}}(x)$ is an upper semi-continuous membership function,

ii) $\tilde{a}$ is a convex fuzzy set, i.e. $\mu_{\tilde{a}}(\lambda x+(1-\lambda) y) \geq \min \left\{\mu_{\tilde{a}}(x), \mu_{\tilde{a}}(y)\right\}$ for all $x, y \in R$ and $0 \leq \lambda \leq 1$,

iii) $\tilde{a}$ is normal, i.e. $\exists x_{0} \in R$ for which $\mu_{\tilde{a}}\left(x_{0}\right)=1$,

iv) $\operatorname{supp}(\tilde{a})=\left\{x \in R: \mu_{\tilde{a}}>0\right\}$ is the support of the $\tilde{a}$ and its closure $\operatorname{cl}(\operatorname{supp}(\tilde{a}))$ is compact set. 
Definition 2. A triangular fuzzy number can be represented completely by a triplet $\tilde{A}=$ $\left(a_{1}, a_{2}, a_{3}\right)$ and has membership:

$$
\mu_{\tilde{A}}(x)=\left\{\begin{array}{cl}
0 & \text { if } x<a_{1}, \\
\frac{x-a_{1}}{a_{2}-a_{1}} & \text { if } a_{1} \leq x \leq a_{2}, \\
\frac{a_{3}-x}{a_{3}-a_{2}} & \text { if } a_{2} \leq x \leq a_{3}, \\
0 & \text { if } x>a_{3} .
\end{array}\right.
$$

Definition 3. A fuzzy number $\tilde{B}$ is said to be $L-R$ type if

$$
\mu_{\tilde{B}}(x)= \begin{cases}L\left(\frac{m-x}{\alpha}\right) & \text { if } x \leq m \text { and } \alpha>0, \\ R\left(\frac{x-m}{\beta}\right) & \text { if } x \geq m \text { and } \beta>0,\end{cases}
$$

where $m$ is the mean value of $\tilde{B}, \alpha$ and $\beta$ are left and right spreads, respectively, and a function $L(\cdot)$ is a left shape function satisfying

i) $L(x)=L(-x)$,

ii) $L(0)=1$,

iii) $L(x)$ is non decreasing on $[0, \infty)$.

Similarly, a right shape function $R(\cdot)$ is defined as $L(\cdot)$. Symbolically, a $L-R$ fuzzy number $\tilde{B}$ can be written as $\tilde{B}=(b, \alpha, \beta)_{L R}$.

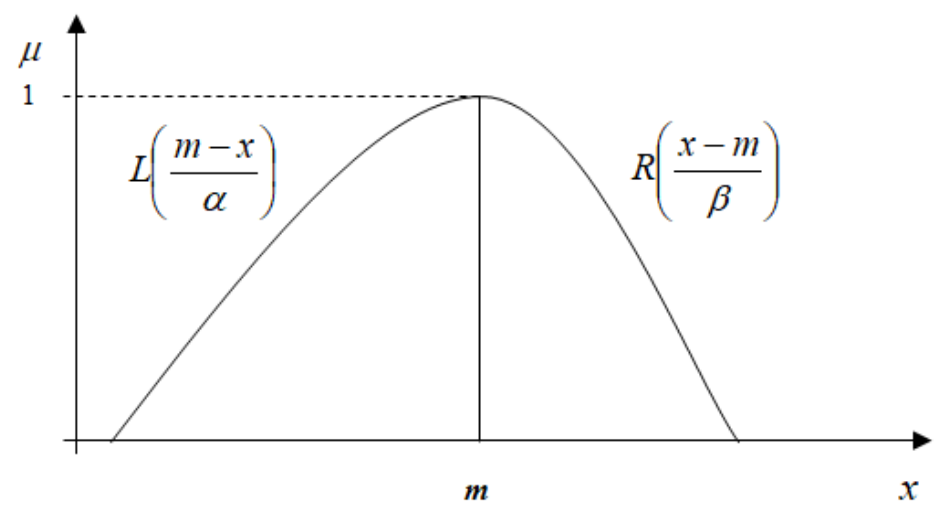

Figure 1: $L-R$ fuzzy number

The formulas of addition, subtraction, opposite, multiplication and order relation for $\tilde{A}=$ $(a, \gamma, \delta)_{L R}$ and $\tilde{B}=(b, \alpha, \beta)_{L R}$ are

- addition $\tilde{A} \oplus \tilde{B}=(a+b, \gamma+\alpha, \delta+\beta)_{L R}$

- opposite $-\tilde{A}=(-a, \gamma, \delta)_{L R}$

- substraction $\tilde{A}(-) \tilde{B}=(a-b, \gamma+\beta, \delta+\alpha)_{L R}$ 
- multiplication $\tilde{A} \otimes \tilde{B}$ :

if $\tilde{A}>0$ and $\tilde{B}>0$ then $\tilde{A} \otimes \tilde{B} \cong(a b, a \alpha+b \gamma, a \beta+b \delta)_{L R}$,

if $\tilde{A}<0$ and $\tilde{B}>0$ then $\tilde{A} \otimes \tilde{B} \cong(a b,-b \delta-a \beta,-b \gamma-a \alpha)_{L R}$,

if $\tilde{A}<0$ and $\tilde{B}<0$ then $\tilde{A} \otimes \tilde{B} \cong(a b, b \gamma-a \beta, b \delta-a \alpha)_{L R}$

- inverse $\tilde{A}^{-1} \cong\left(a^{-1}, \delta a^{-2}, \gamma a^{-2}\right)_{L R}$

- order relation $\tilde{A}(<) \tilde{B}$ iff $a<b$, or $a=b$ and $(\gamma+\delta)>(\alpha+\beta)$ or $a=b,(\gamma+\delta)=$ $(\alpha+\beta)$ and $(2 a-\gamma+\delta)<(2 b-\alpha+\beta)$

\section{Problem formulation and solution concepts}

A fully fuzzy linear programming problem can be formulated as follows

$$
\begin{gathered}
\min \tilde{Z}=\tilde{C}^{T} \otimes \tilde{X} \\
\text { s.t. } \tilde{A} \otimes \tilde{X}=\tilde{B}, \\
\tilde{X} \geq \tilde{0},
\end{gathered}
$$

where $\tilde{C}^{T}=\left(\tilde{c}_{j}\right)_{1 \times n}, \tilde{X}=\left(\tilde{x}_{j}\right)_{n \times 1}, \tilde{A}=\left(\tilde{a}_{i j}\right)_{m \times n}, \tilde{B}=\left(\tilde{b}_{i}\right)_{m \times 1}$ and $\tilde{c}_{j}, \tilde{x}_{j}, \tilde{a}_{i j}, \tilde{b}_{i} \in F_{0}(R)$. Through this paper $F_{0}(R)$ is the set of all $L-R$ fuzzy numbers on $R$.

Definition 4. The $\tilde{x}^{*}$ which satisfies the conditions in problem (1) is called a fuzzy optimization solution [32].

The FFLP problem (1) may be written as

$$
\begin{aligned}
\min \tilde{Z}= & \sum_{j=1}^{n}\left(c_{j}, \alpha_{j}, \beta_{j}\right)_{L R} \otimes\left(x_{j}, \zeta_{j}, \xi_{j}\right)_{L R} \\
\text { s.t. } & \sum_{j=1}^{n}\left(a_{i j}, \varepsilon_{i j}, \phi_{i j}\right)_{L R} \otimes\left(x_{j}, \zeta_{j}, \xi_{j}\right)_{L R}=\left(b_{i}, \mu_{i}, v_{i}\right)_{L R}, \\
& \left(x_{j}, \zeta_{j}, \xi_{j}\right)_{L R} \geq \tilde{0} .
\end{aligned}
$$

Using the arithmetic operations of $L-R$ fuzzy numbers, problem (2) may be rewritten as

$$
\begin{aligned}
\min \tilde{Z}= & \sum_{j=1}^{n}\left(c_{j} x_{j}, c_{j} \zeta_{j}+\alpha_{j} x_{j}, c_{j} \xi_{j}+\beta_{j} x_{j}\right)_{L R} \\
\text { s.t. } & \sum_{j=1}^{n}\left(a_{i j} x_{j}, a_{i j} \zeta_{j}+\varepsilon_{i j} x_{j}, a_{i j} \xi_{j}+\phi_{i j} x_{j}\right)_{L R}=\left(b_{i}, \mu_{i}, v_{i}\right)_{L R}, \\
& \left(x_{j}, \zeta_{j}, \xi_{j}\right)_{L R} \geq \tilde{0} .
\end{aligned}
$$


Also, from problem (3) we have the following problem

$$
\begin{gathered}
\min \tilde{Z}=\sum_{j=1}^{n}\left(\left(c_{j} x_{j}\right)^{L},\left(c_{j} x_{j}\right)^{C},\left(c_{j} x_{j}\right)^{U}\right) \\
\text { s.t. } x \in X=\left\{\begin{array}{l}
\sum_{j=1}^{n} a_{i j} x_{j}=b_{i}, \quad i=1,2, \ldots, m \\
\sum_{j=1}^{n}\left(a_{i j} \zeta_{j}+\varepsilon i j x_{j}, a_{i j} \xi_{j}+\phi_{i j} x_{j}\right)=\mu_{i}+v_{i}, \quad \forall i \\
\sum_{j=1}^{n}\left(2 a_{i j} x_{j}-\left(a_{i j} \zeta_{j}+\varepsilon i j x_{j}\right)+\left(a_{i j} \xi_{j}+\phi_{i j} x_{j}\right)\right)=2 b_{i}-\mu_{i}+v_{i} \quad \forall i \\
x_{j}, \zeta_{j}, \xi_{j} \geq 0 \quad \forall j,
\end{array}\right.
\end{gathered}
$$

where $\left(c_{j} x_{j}\right)^{C}=c_{j} x_{j},\left(c_{j} x_{j}\right)^{L}=c_{j} x_{j}-\left(a_{i j} \zeta_{j}+\varepsilon_{i j} x_{j}\right)$ and $\left(c_{j} x_{j}\right)^{U}=c_{j} x_{j}+\left(c_{j} \xi_{j}+\beta_{j} x_{j}\right)$. From problem (4) we have the following MOLP problem

$$
\begin{aligned}
\operatorname{minf}_{1} & =\left(c_{j} x_{j}\right)^{C} \\
\operatorname{minf}_{2} & =\left(c_{j} x_{j}\right)^{U}-\left(c_{j} x_{j}\right)^{L} \\
\operatorname{minf} f_{3} & =\left(c_{j} x_{j}\right)^{U}+\left(c_{j} x_{j}\right)^{L} \\
\text { s.t. } x & \in X .
\end{aligned}
$$

Definition 5. (Pareto optimal solution) $x^{0} \in X$ is said to be Pereto optimal solution to problem (5) if and only if there does not exist another $x \in X$ such that $f_{1}(x) \leq f_{1}\left(x^{0}\right), f_{2}(x) \geq Z_{2}\left(x^{0}\right)$ and $f_{3}(x) \leq f_{3}\left(x^{0}\right)$, and $f_{1}(x) \neq f_{1}\left(x^{0}\right), f_{2}(x) \geq f_{2}\left(x^{0}\right)$ or $f_{3}(x) \neq f_{3}\left(x^{0}\right)$.

Problem (5) can be treated using the weighting method [20] as

$$
\begin{aligned}
& \min E=\left(w_{1} f_{1}-w_{2} f_{2}+w_{3} f_{3}\right) \\
& \text { s.t. } x \in X, \quad w_{i} \geq 0, \quad \sum_{i=1}^{3} w_{i}=1 .
\end{aligned}
$$

\section{Proposed approach}

The steps of the proposed approach for solving the FFLP problem (1) can be summarized as:

Step 1: Consider the FFLP problem (1)

Step 2: Convert the problem (1) into problem (4)

Step 3: Transform the problem (4) into the MOLP problem (5), and then into problem (6)

Step 4: Solve the problem (6) to obtain the efficient solution

Step 5: Referring to problem (1) for determining the fuzzy optimal solution and the corresponding fuzzy optimum value

Step 6: Stop 


\section{Numerical example}

Consider the following FFLP problem

$$
\begin{array}{ll}
\min \tilde{Z}=(2,1,1)_{L R} \otimes \tilde{x}_{1} \oplus(3,1,1)_{L R} \otimes \tilde{x}_{2} \\
\text { s.t. } & (1,1,1)_{L R} \otimes \tilde{x}_{1} \oplus(2,1,1)_{L R} \otimes \tilde{x}_{2}=(10,8,14)_{L R} \\
& (2,1,1)_{L R} \otimes \tilde{x}_{1} \oplus(1,1,1)_{L R} \otimes \tilde{x}_{2}=(8,7,13)_{L R} \\
& \tilde{x}_{1}, \tilde{x}_{2} \geq 0, \quad \tilde{x}_{1}, \tilde{x}_{2} \in F_{0}(R) .
\end{array}
$$

Based on the proposed approach, the above FFLP problem can be written as

$$
\begin{aligned}
& \min f_{1}=2 x_{1}+3 x_{2} \\
& \min f_{2}=2 x_{1}+2 x_{2}+2 \xi_{1}+3 \xi_{2}+2 \zeta_{1}+3 \zeta_{2} \\
& \min f_{3}=4 x_{1}+6 x_{2}+2 \xi_{1}+3 \xi_{2}-2 \zeta_{1}-3 \zeta_{2} \\
& \text { s.t. } \quad x_{1}+2 x_{2}=10 \\
& \quad 2 x_{1}+2 x_{2}+\xi_{1}+2 \xi_{2}+\zeta_{1}+2 \zeta_{2}=22 \\
& \quad 2 x_{1}+4 x_{2}-\xi_{1}-2 \xi_{2}+\zeta_{1}+2 \zeta_{2}=26 \\
& \quad 2 x_{1}+x_{2}=8 \\
& 2 x_{1}+2 x_{2}+2 \xi_{1}+\xi_{2}+2 \zeta_{1}+\zeta_{2}=20 \\
& 4 x_{1}+2 x_{2}-2 \xi_{1}-\xi_{2}+2 \zeta_{1}+\zeta_{2}=22 \\
& \quad x_{1}, x_{2}, \xi_{1}, \xi_{2}, \zeta_{1}, \zeta_{2} \geq 0 \\
& \quad x_{1}-2 \zeta_{1} \geq 0, x_{1}+2 \zeta_{1} \geq 0, x_{1}+2 \xi_{2} \geq 0 \\
& \quad x_{2}-3 \zeta_{2} \geq 0, x_{2}+3 \zeta_{2} \geq 0, x_{2}+3 \xi_{2} \geq 0 .
\end{aligned}
$$

Using the weighting method, the problem (8) becomes

$$
\begin{aligned}
\min E= & 0.5 f_{1}+0.2 f_{2}+0.3 f_{3}=1.8 x_{1}+2.9 x_{2}+0.2 \xi_{1}+0.3 \xi_{2}-\zeta_{1}-1.5 \zeta_{2} \\
\text { s.t. } & x_{1}+2 x_{2}=10 \\
& 2 x_{1}+2 x_{2}+\xi_{1}+2 \xi_{2}+\zeta_{1}+2 \zeta_{2}=22 \\
& 2 x_{1}+4 x_{2}-\xi_{1}-2 \xi_{2}+\zeta_{1}+2 \zeta_{2}=26 \\
& 2 x_{1}+x_{2}=8 \\
& 2 x_{1}+2 x_{2}+2 \xi_{1}+\xi_{2}+2 \zeta_{1}+\zeta_{2}=20 \\
& 4 x_{1}+2 x_{2}-2 \xi_{1}-\xi_{2}+2 \zeta_{1}+\zeta_{2}=22 \\
& x_{1}, x_{2}, \xi_{1}, \xi_{2}, \zeta_{1}, \zeta_{2} \geq 0 \\
& x_{1}-2 \zeta_{1} \geq 0, x_{1}+2 \zeta_{1} \geq 0, x_{1}+2 \xi_{2} \geq 0 \\
& x_{2}-3 \zeta_{2} \geq 0, x_{2}+3 \zeta_{2} \geq 0, x_{2}+3 \xi_{2} \geq 0 .
\end{aligned}
$$

Using GAMS Software, the solution of FFLP problem (7) and thus the solution of problem (9) is given in Table 1.

\begin{tabular}{|c|c|}
\hline Fuzzy optimal solution & Fuzzy optimum value \\
\hline \hline$\tilde{x}_{1}^{*}=(2,0,2)_{L R}$ & $\tilde{Z}^{*}=(16,9,19)_{L R}$ \\
$\tilde{x}_{2}^{*}=(4,1,3)_{L R}$ & $E=15$ \\
\hline
\end{tabular}

Table 1: The fuzzy solution of the FFLP problem 


\section{Conclusions}

In this paper, an approach for obtaining fuzzy optimal solution for FFLP has introduced. The proposed approach based on converting the FFPL problem into triple objective function and hence single objective using the weighting method. The advantage of this approach is more flexible, realistic to the real world problem, useful for the future study and can be extended in generalized fuzzy numbers.

\section{Acknowledgements}

The author would like to thank the referees for their suggestive valuable and helpful comments that have led to an improved version of the paper.

\section{References}

[1] Allahviranloo, T., Lotfi, F. H., Kiasary, M. K., Kiani, N. A. and Alizadeh, L. (2008). Solving fully fuzzy linear programming problem by the ranking function. Applied Mathematical Sciences, 2(14), 19-32. http://www.m-hikari.com/ams/ams-password-2008/ams-password1-4-2008/index. html

[2] Bector, C. R. and Chandra, S. (2005). Fuzzy Mathematical Programming and Fuzzy Matrix Games. Studies in Fuzzines and Soft Computing. Springer: Berlin. doi: 10.1007/3-540-32371-6

[3] Bellman, R. E. and Zadeh, L. A. (1970). Decision-Making in a Fuzzy Environment. Management Science, 17(4), 141-164. doi: 10.1287/mnsc.17.4.B141

[4] Buckley, J. J. (1988). Possibilistic linear programming with triangular fuzzy numbers. Fuzzy Sets and Systems, 26(1), 135-138. doi: 10.1016/0165-0114(88)90013-9

[5] Buckley, J. J. (1989). Solving possibilistic linear programming problems. Fuzzy Sets and Systems, (31)3, 329-341. doi: 10.1016/0165-0114(89)90204-2

[6] Carlsson, C. and Korhonen, P. (1986). A parametric approach to fuzzy linear programming. Fuzzy Sets and Systems, 20(1), 17-30. doi: 10.1016/s0165-0114(86)80028-8

[7] Chanas, S. (1983). The use of parametric programming in fuzzy linear programming. Fuzzy Sets and Systems, 11(1-3), 243-251. doi: /10.1016/s0165-0114(83)80083-9

[8] Das, S. K. (2017). Modified method for solving fully fuzzy linear programming problem with triangular fuzzy numbers. International Journal of Research in Industrial Engineering, 6(4), 293311. doi: 10.22105/riej.2017.101594.1024

[9] Das, S. K., Mandal, T. and Behera, D. (2019). A new approach for solving fully fuzzy linear programming problem. International Journal of Mathematics in Operational Research, 15(3), 296309. doi: 10.1504/ijmor.2019.10023109

[10] Dubois, D. and Prade, H. (1980). Systems of fuzzy linear constraints. Fuzzy Sets and Systems, 3(1), 37-48. doi: 10.1016/0165-0114(80)90004-4

[11] Ezzati, R., Khorram, R. and Enayati, R. A. (2013). A new algorithms to solve fully fuzzy linear programming problems using the MOLP problem. Applied Mathematical Modelling, 39(12), 31833193. doi: 10.1016/j.apm.2013.03.014

[12] Gong, , Z., Zhao, W. and Liu, K. (2018). A straightforward approach for solving fully fuzzy linear programming problem with $L R$-type fuzzy numbers. Journal of the Operations Research Society of Japan, 61(2), 172-185. doi: 10.15807/jorsj.61.172

[13] Hashemi, S. M., Modarrem, M., Nasrabadi, E., and Nasrabadi, M. M. (2006). Fully fuzzified linear programming, solution and duality. Journal of Intelligence and Fuzzy Systems, 17(3), 253-261. https://content.iospress.com/articles/journal-of-intelligent-and-fuzzy-systems/ ifs00295

[14] Hosseinzadeh, A. and Edalatpanah, A. S. (2016). A New Approach for Solving Fully Fuzzy Linear Programming by Using the Lexicography Method. Advances in Fuzzy Systems, 2016, 1-6. doi: $10.1155 / 2016 / 1538496$ 
[15] Ishibuchi, H. and Tanaka, H. (1990). Multiobjective programming in optimization of the interval objective function. European Journal of Operational Research, 48(2), 219-225. doi: 10.1016/03772217(90)90375-1

[16] Kaufmann, A. and Gupta, M. M. (1988). Fuzzy Mathematical Models in Engineering and Management Science. North-Holland.

[17] Kumar, A., Kaur, J. and Singh, P. (2011). A new method for solving fully fuzzy linear programming problems. Applied Mathematical Modelling, 35(12), 817-823. doi: 10.1016/j.apm.2010.07.037

[18] Li, X. and Shi, K. (1998). Fuzzy Linear Programming Problems with Interval-value Fuzzy Coefficients. The Journal of Fuzzy Mathematics, 6(2), 327-332. https://www.tib.eu/en/search/id/ olc\%3A1580365418/Fuzzy-Linear-Programming-Problems-with-Interval/

[19] Maleki, H. R., Tata, M. and Mashinchi, M. (2000). Linear programming with fuzzy variables. Fuzzy Sets and Systems, 109(1), 21-33. doi: 10.1016/s0165-0114(98)00066-9

[20] Miettinen, K. (1998). Nonlinear Multiobjective Optimization. International Series in Operations Research and Management Science, 12. Springer: Boston doi: 10.1007/978-1-4615-5563-6

[21] Ramik, J. and Imanek, J. (1985). Inequality relation between fuzzy numbers and its use in fuzzy optimization. Fuzzy Sets and Systems, 16(2), 123-138. doi: 10.1016/s0165-0114(85)80013-0

[22] Rajarajeswari, P. and Sahaya, S. A. (2014). Solving a Fully Fuzzy Linear Programming Problem by Ranking. International Journal of Mathematics Trends and Technology, 9(2), 159-164. doi: 10.14445/22315373/ijmtt-v9p519

[23] Sahaya, S. A. and Karpagamani, V. (2014). Solving fully fuzzy linear programming problem using trapezoidal ranking function. Journal of Global Research in Mathematical Archives, 2(6), 21-26. http://www.jgrma.info/index.php/jgrma/article/view/231

[24] Sakawa, M. and Yano, H. (1991). Feasibility and Pareto optimality for multiobjective linear programming problems with fuzzy parameters. Fuzzy Sets and Systems, 43(1), 1-15. doi: 10.1016/0165-0114(91)90017-k

[25] Sakawa, M. (1993). Fuzzy Sets and Interactive Multiobjective Optimization. Applied Informatioin Technology. Springer: Boston. doi: 10.1007/978-1-4899-1633-4

[26] Shamooshaki, M. M., Hosseinzadeh, A. and Edalatpanah, S. A. (2015). A New Method for Solving Fully Fuzzy Linear Programming Problems by Using the Lexicography Method. Applied and Computational Mathematics, 4(1-2), 1-3. doi: 10.11648/j.acm.s.2015040102.11

[27] Shaocheng, T. (1994). Interval number and fuzzy number linear programming. Fuzzy Sets and Systems, 66(3), 301-306. doi: 10.1016/0165-0114(94)90097-3

[28] Tanaka, H., Okuda, T. and Asai, K. (1974). On Fuzzy-Mathematical Programming. Journal of Cybernetics, 3(4), 37-46. doi: 10.1080/01969727308545912

[29] Tanaka, H., Ichihashi, H. and Asai, K. (1984). A formulation of fuzzy linear programming problem based on comparison of fuzzy number. Control of Cybernetics, 13(3), 185-194. http://control. ibspan. waw .pl:3000/contents/show/135?year=1984

[30] Zadeh, L. A. (1965). Fuzzy sets. Information and Control, 8(3), 338-353. doi: 10.1016/s00199958(65)90241-x

[31] Zhao, R., Govind, R. and Fan, G. (1992). The complete decision set of the generalized symmetrical fuzzy linear programming problem. Fuzzy Sets and Systems, 51(1), 53-65. doi: 10.1016/01650114(92)90075-f

[32] Zhong, Q., Yue, Z. and Guangyuan, W. (1994). On fuzzy random linear programming. Fuzzy Sets and Systems, 65(1), 31-49. doi: 10.1016/0165-0114(94)90245-3

[33] Zimmermann, H. J. (1978). Fuzzy programming and linear programming with several objective functions. Fuzzy Sets and Systems, 1(1), 45-55. doi: 10.1016/0165-0114(78)90031-3 